\title{
The Locus Ceruleus Responds to Signaling Molecules Obtained from the CSF by Transfer through Tanycytes
}

\author{
Cheng-Yuan Feng, Larisa M. Wiggins, and Christopher S. von Bartheld \\ Department of Physiology and Cell Biology, University of Nevada School of Medicine, Reno, Nevada 89557
}

\begin{abstract}
Neurons can access signaling molecules through two principal pathways: synaptic transmission ("wiring transmission") and nonsynaptic transmission ("volume transmission"). Wiring transmission is usually considered the far more important mode of neuronal signaling. Using embryonic chick locus ceruleus (LoC) as a model, we quantified and compared routes of delivery of the neurotrophin nerve growth factor (NGF), either through a multisynaptic axonal pathway or via the CSF. We now show that the axonal pathway from the eye to the LoC involves axo-axonic transfer of NGF with receptor switching (p75 to trkA) in the optic tectum. In addition to the axonal pathway, the LoC of chick embryos has privileged access to the CSF through a specialized glial/ependymal cell type, the tanycyte. The avian LoC internalizes from the CSF in a highly specific fashion both NGF and the hormone urotensin (corticotropin-releasing factor family ligand). Quantitative autoradiography at the ultrastructural level shows that tanycytes transcytose and deliver NGF to LoC neurons via synaptoid contacts. The LoC-associated tanycytes express both p75 and trkA receptors. The NGF extracted by tanycytes from the CSF has physiological effects on LoC neurons, as evidenced by significantly altered nuclear diameters in both gain-of-function and loss-of-function experiments. Quantification of NGF extraction shows that, compared with multisynaptic axonal routes of NGF trafficking to LoC, the tanycyte route is significantly more effective. We conclude that some clinically important neuronal populations such as the LoC can use a highly efficient "back door" interface to the CSF and can receive signals via this tanycyte-controlled pathway.
\end{abstract}

\section{Introduction}

Neurons receive signals by two main pathways: synaptic contacts ("wiring transmission") as well as systemic flow ("volume transmission") (Agnati et al., 1995). Neurons in the brain receive information primarily via axonal transport and synaptic transmission, but they can also respond to signaling molecules in the CSF (Lehman and Silver, 2000). Because the ependymal lining of the ventricles forms a CSF-brain barrier (Del Bigio, 1995; Bruni, 1998), the role of CSF as a source of signaling molecules has been unclear (Nicholson, 1999). Multiple sources and types of tissues release signaling molecules into the CSF (Vigh and Vigh-Teichmann, 1998; Hochhaus et al., 2001; Mashayekhi et al., 2009), but the fate and significance of such molecules has remained controversial.

A collection of noradrenergic neurons in the brainstem, the locus ceruleus (LoC), is the primary source of noradrenaline in the brain and integrates the brain's stress response among other functions (Berridge and Waterhouse, 2003; Gonzalez and AstonJones, 2006; Valentino and Van Bockstaele, 2008). These neurons

Received Sept. 23, 2010; revised May 2, 2011; accepted May 4, 2011.

Author contributions: C.-Y.F. and C.S.v.B. designed research; C.-Y.F., L.M.W., and C.S.v.B. performed research; C.-Y.F., L.M.W., and C.S.v.B. analyzed data; C.-Y.F. and C.S.v.B. wrote the paper.

This work was supported by NIH Grants HD 29177 and EY 12841 and NCRR Grants P20 RR 15581 (Center of Biomedical Research Excellence) and P20 RR 016464 (Nevada IDeA Network of Biomedical Research Excellence). We thank Andreas Schober (University of Heidelberg, Germany) and Will Hatton and Honglin Tian (University of Nevada) for sharing their expertise.

Correspondence should be addressed to Christopher S. von Bartheld, Department of Physiology and Cell Biology, Mailstop 352, University of Nevada School of Medicine, Reno, NV 89557. E-mail: cvonbartheld@medicine.nevada.edu.

DOI:10.1523/JNEUROSCI.5018-10.2011

Copyright $\odot 2011$ the authors $\quad 0270-6474 / 11 / 319147-12 \$ 15.00 / 0$ can be activated by axonally transported neurotransmitters and neuropeptides (Valentino and Van Bockstaele, 2008), including members of the corticotropin-releasing factor (CRF) family. Since neurons expressing relevant receptors accumulate and respond to both axon-derived as well as CSF-derived signals, contributions of signaling molecules from different sources and their pathways to the LoC have remained unclear.

In the avian brain, the noradrenergic neurons of the LoC express trkA, the specific receptor for nerve growth factor (NGF), and these neurons are regulated by NGF (von Bartheld et al., 1995). One of the richest sources of endogenous NGF is the eye (Large et al., 1989; Lambiase et al., 2002), prompting the question whether periphery-derived NGF may gain access to the LoC. Here we report the results of a comprehensive trafficking study of nerve growth factor and urotensin, a CRF family member. By quantifying and comparing trafficking routes, we show that LoC neurons can receive NGF not only by axonal transport via multisynaptic, axo-axonic synapses, but also by extracting (very efficiently) NGF from the CSF via a novel pathway, by transcytosis through CSF-contacting tanycytes. This privileged access of the LoC to the CSF is remarkably selective, as it is used by only a small number of molecules: NGF and urotensin-1 (a CRF family ligand), but not neurotrophin-3 (NT-3), fibroblast growth factor 2 (FGF2), or glial cell line-derived neurotrophic factor (GDNF). Remarkably, we show that the nuclear diameter of LoC neurons-a measure of LoC neuronal activity (Bubenik and Monnier, 1972; Smialowska et al., 1988)—is controlled by the amount of endogenous and exogenous NGF in the CSF. Our data reveal and characterize a novel interface of communication between the 
LoC and the CSF, with potentially wide-ranging physiological and clinical implications.

\section{Materials and Methods}

Animals. Fertilized chicken eggs (White Leghorn) were obtained from a local supplier and incubated in a force-draft incubator at $37.5^{\circ} \mathrm{C}$. A total of $\sim 1550$ chick embryos of both sexes were used. All experimental procedures were approved by the local animal care committee and were conducted in compliance with the Policy on the Use of Animals in Neuroscience Research (Society for Neuroscience).

Materials. Murine NGF was from Alomone Labs or Promega. Human recombinant BDNF and NT-3 were kindly provided by Regeneron. GDNF and FGF2 were from PeproTech, and urotensin-1 was from American Peptide. Polyclonal antibodies against chicken p75 or chicken trk receptors were gifts from Louis Reichardt (University of California, San Francisco, San Fancisco), and monoclonal antibody M7412 against chicken p75 was a gift from Hideaki Tanaka (Kumamoto University, Kumamoto, Japan). Rabbit anti-p75 antibody against the intracellular domain was from Promega. Rabbit anti-tyrosine hydroxylase (TH) antibodies were from Millipore. Rabbit polyclonal NGF antibody was from Abcam. Cytochrome C, colchicine, monensin, and 6-OHDA were from Sigma, and tetanus toxin C fragment was from Calbiochem. Streptavidin Alexa Fluor 488 conjugate was from Invitrogen. PVDF membrane, goatanti-rabbit secondary antibody conjugated to alkaline phosphatase (AP), and AP conjugate substrate kit were from Bio-Rad.

Iodination, injections, and processing for autoradiography. Neurotrophins were radiolabeled with ${ }^{125}$ using lactoperoxidase, as described previously (von Bartheld, 2001). Incorporations were typically $85-95 \%$, except for cytochrome C (50-65\%). Specific activities were 61-100 cpm/pg NGF, 71-197 cpm/pg NT-3, 29-94 cpm/pg GDNF, 110 cpm/pg FGF2, $49 \mathrm{cpm} / \mathrm{pg}$ urotensin-1, 42-119 cpm/pg cytochrome C, and 73 $\mathrm{cpm} / \mathrm{pg}$ tetanus toxin $\mathrm{C}$ fragment. Neurotrophins were used within 1 week after iodination; bioactivity was preserved within this time frame. Autoradiography and quantification of radiolabeled proteins were performed as described previously (von Bartheld et al., 1996a,b; von Bartheld, 2001). Silver grain densities were measured either by dividing the percentage of silver grains by the percentage of fractional area (labeling density), or by measuring grains per given unit area. Using a $40 \times$ objective, silver grains within $400 \mu \mathrm{m}^{2}$ grids were counted by using a reticule in the eyepiece. For competition experiments, 50- to 80-fold excess cold NGF was coinjected with $20 \mathrm{ng}$ of radiolabeled NGF $(n=4)$, and silver grains were counted per $400 \mu \mathrm{m}^{2}$, normalized by dividing the counts per minute times $10^{-6}$ in the eye at the time of death. For silver grain accumulation in the tectum over time, 10 time points $(2,4,7,9,18,20,28,41$, 54 , and $64 \mathrm{~h}$ after eye injection) were examined. Accumulation was scored semiquantitatively with values between 0 and 4 , blind to time point, and plotted as a function of time after intraocular injection.

Quantitative ultrastructural autoradiography. Sections through the optic tectum and the LoC were analyzed by ultrastructural autoradiography using protocols described previously (von Bartheld et al., 1996a; Butowt and von Bartheld, 2001; von Bartheld, 2001). In short, tissues were perfusion fixed with $2 \%$ glutaraldehyde and $2 \%$ paraformaldehyde (PFA), dissected, fixed in osmium tetroxide, and embedded in Spurr's resin. Using the "loop method," thin sections $(70-80 \mathrm{~nm})$ on copper grids were coated with a monolayer of Ilford L4 emulsion and exposed in the dark for 2-3 months. Silver grains were developed, sections were lightly stained with lead citrate, and silver grains were observed on a Philips CM10 transmission electron microscope. Images were photographed on film $(8.3 \times 10.2 \mathrm{~cm}$ Kodak EM Film $)$ or digital images captured on a Gatan Bioscan 792 imaging system. Silver grains were quantified by measuring labeling densities over particular cell types or organelles. For the determination of labeling densities in the ependyma, neuropil, and LoC, a total area of $31,500 \mu \mathrm{m}^{2}$ was examined. A total of 125 images were acquired, and 1282 silver grains were analyzed for these experiments. For silver grain analysis in the tectum, at least 100 silver grains were analyzed for each condition and time point $(4,20$, and $54 \mathrm{~h}$ after eye injection).

Electron microscopy of the fourth ventricle ependyma adjacent to the locus ceruleus. To determine whether LoC neurons may send processes through the ependyma into the CSF, as is known for other types of neurons in the third ventricle of some species (von Bartheld and Meyer, 1990), we performed an exhaustive search of the ependyma adjacent to the LoC. Overall, the ependyma was examined in 23 animals, with a total of 30 tissue blocks sampled. The area of interest was first identified in semithin $(1 \mu \mathrm{m})$ sections perpendicular to the long axis of the ependyma, spaced $\sim 15 \mu \mathrm{m}$ apart (a total of 75 semithin sections), and then a series of thin sections $(80-100 \mathrm{~nm}$ ) was collected from each site, overall $\sim 900$ thin sections. A total of 352 grids were collected and stained successfully, which allowed the scanning of a total area of $350,000 \mu \mathrm{m}^{2}$ within the ependyma of interest.

Cross-linking and immunoprecipitation. Chick embryos at embryonic day 15 (E15) to E17 were injected with 3-10 $\mu \mathrm{l}$ of radiolabeled NGF (100-300 ng) in the eye in ovo. Twenty hours later, the animals were anesthetized with Nembutal and transcardially perfused with cold PBS. The optic chiasm, the tectum, and the isthmo-optic nucleus (ION) were dissected, pooled, lysed, and NGF was cross-linked with disuccinimidyl suberate or 1-ethyl-3 (3-dimethylaminopropyl) carbodiimide-HCl (Pierce). For immunoprecipitation (IP), we used polyclonal antibodies specific for chicken neurotrophin receptors and Pansorbin cells (Calbiochem) as described previously (Butowt and von Bartheld, 2001). All analyses were performed in triplicate, and with each set of samples from three to five independent experiments.

6-Hydroxydopamine injections. In 39 chick embryos, freshly prepared 6-OHDA was injected to eliminate chick locus ceruleus fibers (von Bartheld et al., 1995). After windowing, the heads were held in place with sterile surgical forceps, and $150 \mu \mathrm{g}$ of 6-OHDA was injected into the center of each hemisphere of the forebrain using an insulin syringe as described previously (von Bartheld et al., 1995). The needle tip was coated with DiI dissolved in $100 \%$ ethanol to verify the injection site at the time the embryo was killed. The midbrains of 10 embryos were processed 10-24 h later for tyrosine hydroxylase immunofluorescence, while 29 embryos were injected intraocularly with radiolabeled NGF and processed for IP as described above.

TH immunofluorescence. Standard immunocytochemistry procedures were applied on sections from paraffin-embedded brains. Sections were treated with trypsin $(1 \mathrm{mg} / \mathrm{ml})$ for $2-4 \mathrm{~min}$. The $\mathrm{TH}$ primary antibody was used at 1:250 dilution. Control sections for each antiserum were incubated without the primary antibodies. Biotinylated goat-anti-rabbit IgG was used ( $1 \mathrm{~h} ; 2 \mu \mathrm{g} / \mathrm{ml})$, followed by streptavidin Alexa Fluor 488 conjugate at $5 \mu \mathrm{g} / \mathrm{ml}$ for $1 \mathrm{~h}$. Sections were observed on a Nikon Eclipse (E600) microscope and images captured on a Nikon camera (Coolpix 4300) and processed in Adobe Photoshop for contrast and brightness.

SDS-PAGE analysis. For SDS gel analysis, the LoC was dissected from animals injected with ${ }^{125}$ I-NGF in the eyes. Tissues were pooled, homogenized in detergent lysis buffer, centrifuged, and gamma counted, and samples of the supernatant were loaded onto $15 \%$ SDS polyacrylamide gels. Gels were stained with Coomassie blue, dried, and exposed to x-ray film for 2-4 months as described previously (von Bartheld et al., 1996a; Butowt and von Bartheld, 2001).

NGF receptor immunocytochemistry. For immunocytochemical analysis, we used monoclonal (M7412) and polyclonal antibodies against chicken p75, or chicken trkA, using protocols described previously (von Bartheld et al., 1995). Sections were processed either free floating (for EM analysis) or "on the slide." Biotinylated secondary antibodies were visualized with HRP-conjugated streptavidin and the chromogen diaminobenzidine (DAB). For EM analysis, the LoC region was microdissected from 80 to $100 \mu \mathrm{m}$ free-floating sections after DAB reaction and osmicated, and thin sections were cut perpendicular to the ependymal layer and stained with lead citrate and uranyl acetate. In addition, antibodies against TH were used with streptavidin Alexa Fluor 488 conjugate for visualization of fluorescence at the light microscopic level. Control reactions were performed with omission of the primary antibody.

Catecholamine autofluorescence. For double labeling and to examine accumulation of radiolabeled proteins in LoC neurons, we performed either TH immunolabeling or formaldehyde-induced catecholamine fluorescence histochemistry on paraffin sections (Felten et al., 1981b). Adjacent sections were monoreacted to evaluate any potential quenching.

Neurotrophin dot blot assay. NGF, BDNF, and NT-3 were blotted onto $0.2 \mu \mathrm{m}$ PVDF membranes, blocked in $0.5 \%$ gelatin in TNT buffer $(10 \mathrm{~mm}$ 
Tris- $\mathrm{HCl}, \mathrm{pH}$ 8.0, $150 \mathrm{~mm} \mathrm{NaCl}$, and 0.05\% TWEEN 20), and incubated with rabbit polyclonal NGF antibody (1:500). The secondary antibody was goat-anti-rabbit AP conjugated (1:2000), and blots were visualized with Bio-Rad AP conjugate substrate kit.

NGF immunofluorescence. Chicken brain sections of $12 \mu \mathrm{m}$ were treated with $1 \mathrm{mg} / \mathrm{ml}$ trypsin for $3 \mathrm{~min}$ and then incubated with the primary antibody (1:100) in $0.1 \%$ Triton X-100 overnight; the sections were incubated with biotinylated goat-anti-rabbit secondary antibody (1:500) followed by streptavidin Alexa Fluor 488 conjugate (1:100). Sections were imaged on a Nikon Radiance 2100 confocal microscope. Sections without the primary antibody were used as a negative control.

NGF and anti-NGF antibody intraventricular injection and tissue processing. A mixture was prepared containing either PBS (vehicle control), NGF $(20.8 \mathrm{ng} / \mu \mathrm{l})$, or anti-NGF polycolonal antibody $(0.8 \mu \mathrm{g} / \mu \mathrm{l})$ together with GDNF-I ${ }^{125}(15 \mathrm{ng} / \mu \mathrm{l})$ and trypan blue $(0.32 \mu \mathrm{g} / \mu \mathrm{l}$ mixture $)$. Three microliters of this mixture were injected into the tectal ventricle of 11-d-old chick embryos; a second injection was made in the contralateral tectal ventricle $24 \mathrm{~h}$ after the first injection. The dye and the radiolabeled GDNF were added to verify successful injections before quantitative analyses. Forty-eight hours after the first injection, the embryos were perfused intracardially with cold PBS and then 4\% PFA. Ages of chick embryos were verified by measuring third toe length. Successful injections stained the entire ventricular system blue. Brains were embedded in paraffin and sectioned at $12 \mu \mathrm{m}$ for histological analysis. Adjacent sections were collected in two series. One series was used for autoradiography. Since radiolabeled GDNF specifically accumulated at the ventral ependyma exclusively in the ventral midline, but never in or near the LoC, robust radiolabel of GDNF-I ${ }^{125}$ in the midline of the third and fourth ventricles was taken as additional evidence for a successful injection. The other series of sections was processed for tyrosine hydroxylase immunocytochemistry. Images were examined on an upright fluorescent microscope (Nikon Eclipse E600) equipped with a digital camera. In the LoC proper and in the nucleus subceruleus (as a control), the nuclear sizes of neurons containing nucleoli were measured and analyzed with SimplePCI imaging software, blind to treatment to eliminate observer bias.

Karyometric analysis: time course study. To determine how rapidly nuclear size of LoC neurons changed with either diminished or augmented NGF levels in the CSF, chick embryos were examined 4 and $24 \mathrm{~h}$ after injections and compared with the $48 \mathrm{~h}$ time point. As described above, 11-d-old embryos were injected with PBS, NGF, or anti-NGF antibodies into the tectal ventricle. Trypan blue coinjection was deemed sufficient to identify successful injections. Nuclear sizes were measured as described above and compared between the three different time intervals [for control LoC $(n=6),+\operatorname{NGF} \operatorname{LoC}(n=4)$, and $-\operatorname{NGF} \operatorname{LoC}(n=4)]$ for each of the three time points, and statistical significance was analyzed by oneway ANOVA.

In situ hybridization. In situ hybridization for chicken $\mathrm{p} 75$ receptor was performed with a ${ }^{35} \mathrm{~S}$-labeled riboprobe that hybridizes to a $436 \mathrm{bp}$ fragment of chicken p75 receptor mRNA as described previously (von Bartheld and Bothwell, 1992). For detection of trkA expression, ${ }^{35} \mathrm{~S}$-labeled oligonucleotide probes complementary to chicken trkA mRNA were used (von Bartheld et al., 1995). We hybridized paraffin sections (10-12 $\mu \mathrm{m})$ for detection of p75 expression, and frozen sections $(10 \mu \mathrm{m})$ for trkA expression. Sections were lightly counterstained and examined under dark-field and bright-field illumination. Representative images were digitized and are shown without alterations except for optimization of contrast and brightness. Control hybridizations were performed with sense strands.

Neurotrophin tracking by gamma counting. Eyes were gamma counted at the time each embryo was killed. The ION, LoC, and optic tectum were dissected and gamma counted $(n=5-8)$. Samples of CSF were collected and gamma counted, the total CSF volume estimated, and the total amount of radiolabeled NGF in the CSF calculated. Transport and extraction efficiencies along the axonal route as well as the CSF route were calculated and compared. Radiolabeled proteins were recovered from CSF and precipitated with trichloroacetic acid (von Bartheld, 2001). Only the pelleted fraction was used for tracking of NGF. The gamma counts in the supernatant were considered degradation products. Neu- rotrophins that trafficked from the eye to the LoC and tectum were considered largely intact proteins based on SDS-PAGE analysis (von Bartheld et al., 1996a).

Statistical analyses. For axonal transport inhibition experiments and LoC neuron karyometric studies, data from each group passed the normality and equal variance tests. Statistic analyses were performed using one-way ANOVA (multiple comparisons vs control group). For the immunoprecipitation experiments (antibodies against p75 or trkA), unpaired two-tailed $t$ test was used. In all cases, $p<0.05$ was considered statistically significant.

\section{Results}

Eye-derived NGF accumulates in the LoC

The avian LoC expresses the NGF receptors p75 and trkA and responds to NGF (von Bartheld et al., 1995). Sources of endogenous NGF for the avian LoC are believed to include the forebrain and the brainstem (von Bartheld et al., 1995), but by far the richest source of endogenous NGF in the embryonic and adult chicken nervous system is the retina (Large et al., 1989). To determine whether eye-derived NGF may reach the LoC, radiolabeled NGF was injected in the eye of 15- to 17-d-old chicken embryos. Trafficked NGF was visualized in brain sections by autoradiography. Radiolabeled NGF accumulated after $4-28 \mathrm{~h}$ bilaterally in the LoC (Fig. $1 A, B$ ), in addition to the optic nerve and the optic tract, and on the contralateral side, in the optic tectum (Fig. $1 E$ ), isthmo-optic nucleus, and the lateral geniculate nucleus (data not shown). In the LoC, the majority ( $~ 55 \%)$ of noradrenergic neurons were labeled above background, when compared with sections labeled with dopamine- $\beta$-hydroxylase antibodies (von Bartheld and Bothwell, 1992). No other structures in the brain contained eye-derived NGF, and no other molecules tested (NT-3, cytochrome C, tetanus toxin C fragment, or free iodine) accumulated in the LoC after intraocular injections. Quantification showed that silver grains representing radiolabeled NGF in the LoC were 25- to 30 -fold above background, whereas no specific accumulation was observed in the subceruleus neurons (Fig. 1D). The LoC-accumulated NGF was indistinguishable from native NGF when recovered from the LoC and examined by SDS-PAGE (Fig. 1C). Together, these data suggest that eye-derived NGF can reach the LoC specifically and in a bioactive form.

\section{P75 and trkA receptors are sequentially involved in axonal transport of NGF}

We first determined that axonal transport of radiolabeled NGF to the tectum was receptor mediated (reduced by excess "cold" NGF) by quantifying silver grain densities over tectal layers (data not shown). Cross-linking and immunoprecipitation analyses further revealed that NGF bound exclusively to p75 receptors during anterograde transport in retinal axons, but a significant shift occurred in the tectum with nearly $30 \%$ trkA binding (Fig. $1 F$ ). This trkA protein apparently derived from the LoC, since no other neuronal populations in the chicken brain express trkA (von Bartheld et al., 1996b; von Bartheld and Fritzsch, 2006). To verify that the NGF-binding trkA was indeed derived from the LoC, the toxin 6-OHDA was injected into the forebrain, as this causes degeneration of the LoC neurons (von Bartheld et al., 1995). The 6-OHDA treatment resulted in the loss of catecholaminergic fibers in the tectum (Fig. $1 G-I$ ), apparently caused by degeneration of LoC neurons, and indeed a significant reduction of NGF binding to trkA was observed when binding of radiolabeled NGF was quantified by immunoprecipitation in the tectum after intraocular injections of the radiolabeled NGF (Fig. $1 \mathrm{~J})$. Together, these results demonstrate a sequential binding of 

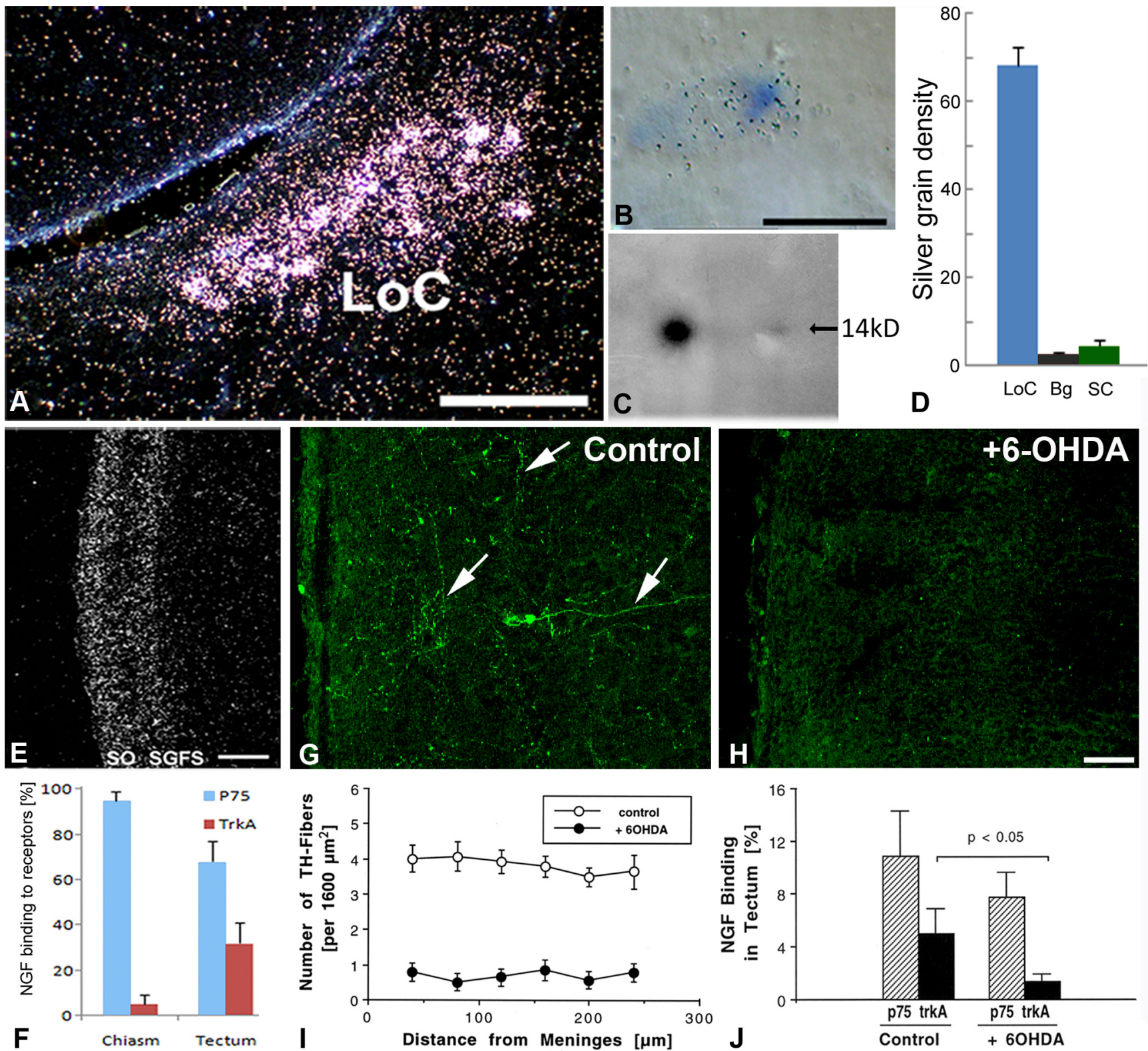

Figure 1. Accumulation of NGF in the LoC after intraocular injection: relay of the axonal pathway in the optic tectum. $A$, Dark-field image showing accumulation of radiolabeled NGF in the LoC of an 18-d-old chick embryo $28 \mathrm{~h}$ after intraocular injection. $\boldsymbol{B}$, Silver grains over LoC cell bodies are shown at higher magnification. $\boldsymbol{C}$, After injection in the eye, radiolabeled NGF was recovered from the LoC intact by SDS-PAGE. Left lane, Native radiolabeled NGF. Right lane, Recovered NGF at 14 kDa. D, Quantification of NGF's labeling density in LoC neurons, compared with background (Bg) and subceruleus neurons (SC). $n=5$. E, NGF accumulated in the superficial layers of the optic tectum where axon terminals (input) from the LoC and RGCs are known to overlap. SGFS, Stratum griseum

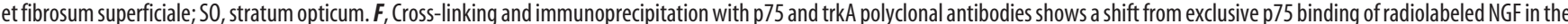
chiasm to substantial trkA binding in the optic tectum. This provides evidence for transfer of NGF from p75-expressing RGC axons to trkA-expressing LoC axons (unpaired two-tailed $t$ test; $n=9$ ). $\boldsymbol{G}$, Catecholaminergic fibers in the superficial layers of the tectum were heavily labeled with TH antibody (arrows). $\boldsymbol{H}$, After 6-OHDA injection in the forebrain, nearly all TH-positive fibers were eliminated in the superficial layers of the tectum. $I$, Quantification of TH-positive fibers in the superficial layers of the tectum in the brains with or without 6-OHDA treatment. The number of TH-positive fibers was reduced by $80-90 \%$ with 6-OHDA treatment. $n=10$.J, Following intraocular injection, I ${ }^{125}$-NGF was immunoprecipitated in the tectum with a trkA antibody. The fraction of trkA-binding NGF was significantly reduced after 6-OHDA treatment compared to controls (unpaired two-tailed $t$ test; $n=4$ independent experiments). The binding of NGF to the $p 75$ receptor was also reduced, presumably because the LoC neurons also express p 75 receptors. Error bars indicate SEM. Scale bars: $\boldsymbol{A}, 200 \mu \mathrm{m} ; \boldsymbol{B}, \boldsymbol{H}, 50 \mu \mathrm{m} ; \boldsymbol{E}, 100 \mu \mathrm{m}$.

NGF to receptors during trafficking, first to p75 alone [in retinal ganglion cell (RGC) axons], and subsequently to trkA and/or a trkA-p75 complex (in LoC axons).

An axonal route to the LoC via axo-axonic synapses in a tectal relay

LoC axons innervate the same layers in the tectum where axons from RGCs terminate (Rodman and Karten, 1995). Approximately $10-20 \%$ of RGC terminals contribute to axo-axonic syn- apses in the retinorecipient avian tectum (Angaut and Reperant, 1976; Wang et al., 2003), and $\sim 30 \%$ of tyrosine-hydroxylasecontaining, presumably noradrenergic terminals from the LoC form axo-axonic synapses in the superior colliculus (Arce et al., 1994). This suggests that NGF may be transported to the LoC after uptake by RGCs (Butowt and von Bartheld, 2001) and via subsequent relay in the optic tectum. We first examined the time course of NGF accumulation in the superficial layers of the tectum. At the light microscopic level, we found that eye-applied 

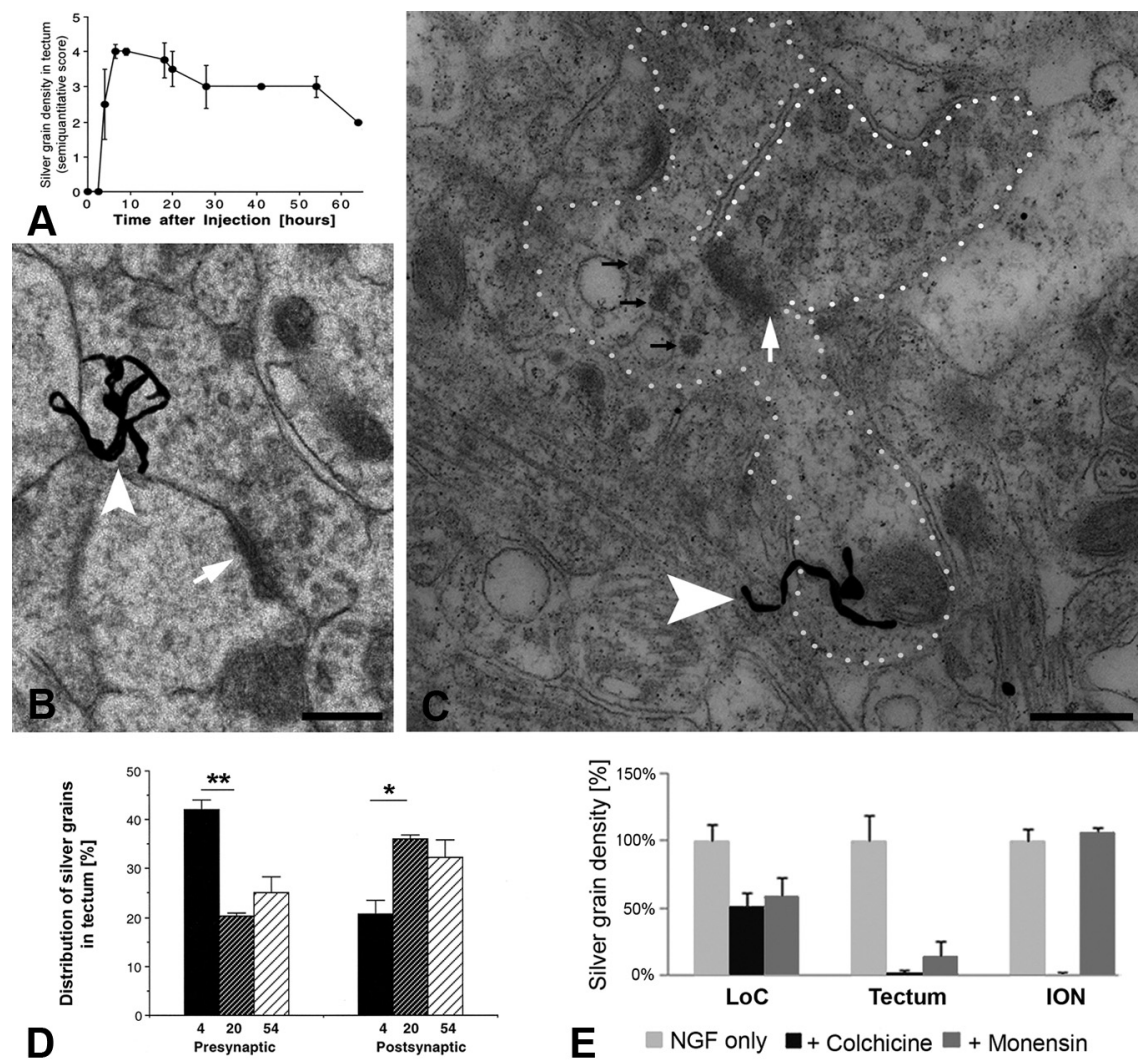

Figure 2. Radiolabeled NGF in axo-axonic tectal synapses and evidence for an additional, nonaxonal pathway from the eye to the LOC. $\boldsymbol{A}$, Time course of accumulation of radiolabeled NGF in the optic tectum. Accumulation was scored semiquantitatively with values between 0 and 4 , blind to time point, and plotted as a function of time after intraocular injection. The mean and SEM are shown. $N=1-5$ per time point. $\boldsymbol{B}$, Ultrastructural autoradiography shows example of radiolabeled NGF in a tectal synapse (arrow). A presumptive retinal ganglion cell axon terminal contains a silver grain (arrowhead) indicative of NGF radiolabeled presynaptically. Such presynaptic silver grains were most abundant $(\sim 40 \%)$ at the $4 \mathrm{~h}$ time point (see $\boldsymbol{D}$ for quantification). $\boldsymbol{C}$ Another presumptive RGC terminal (outlined with white dots, upper right) makes an axo-axonic contact with a presumptive $L O C$ axon terminal (outlined with white dots, left and bottom) that contains several large dense-cored vesicles (black arrows) and a silver grain representing radiolabeled NGF (white arrowhead). Postsynaptic silver grains were most abundant at the $20 \mathrm{~h}$ time point $(\boldsymbol{D})$. Note the synaptic density between the two terminals (white arrow). $\boldsymbol{D}$, Our quantitative autoradiographic time course study shows a significant shift in accumulation of radiolabeled NGF from presynaptic (terminal) to postsynaptic (mostly dendritic) structures in the tectal neuropil from 4 to $54 \mathrm{~h}$ after intraocular injection. Categories sampled included terminal, dendrite, glia, axon, and "not identified." Only the first two categories ( $~ 60-65 \%$ of all silver grains) are shown. ${ }^{*} p=0.025 ;{ }^{* *} p=0.005$, unpaired two-tailed $t$ test. $\boldsymbol{E}$, Elimination of axonal transport in the optic nerve with either colchicine or monensin reduced accumulation of radiolabeled NGF in the LoC by only $40-50 \%$, providing evidence for a major route of NGF to the $L 0 C$ that does not require axonal transport in the optic nerve. Elimination of axonal transport in the optic nerve was confirmed by lack of NGF accumulation in the tectum (anterograde transport) and in the ION (retrograde transport). Error bars indicate SEM. $n=5$. For $p$ values, see Results. Scale bars: $\boldsymbol{B}, \boldsymbol{C}, 500 \mathrm{~nm}$.

NGF appeared in the tectum at $\sim 4 \mathrm{~h}$, and accumulation peaked between 7 and $20 \mathrm{~h}$ and slowly declined thereafter (Fig. $2 \mathrm{~A}$ ). If NGF used an axonal pathway via the tectum, one would expect to find radiolabeled NGF not only in RGC axon terminals in the tectum, but also in axo-axonic synapses in the superficial tectal layers where the retinal input and the LoC input overlap (Rodman and Karten, 1995). Ultrastructural autoradiography showed numerous silver grains in presynaptic synapses (Fig. $2 B, D$ ), and at $20 \mathrm{~h}$, indeed postsynaptic radiolabeled NGF was documented in axo-axonic synapses in the superficial layers of the optic tectum (Fig. 2C). Some of the labeled axo-axonic synapses contained radiolabeled NGF as well as large dense-cored vesicles, indicative of catecholaminergic terminals (Peters et al., 1991; Arce et al., 1994) (Fig. 2C). Quantitative ultrastructural autoradiography of different time points after injection revealed that accumulation of radiolabeled NGF shifted significantly from presynaptic (axon terminals) to postsynaptic (mostly dendritic) sites between 4 and $20 \mathrm{~h}$ after intraocular injection (Fig. 2D). These data, together with our immunoprecipitation and neurotoxin experiments (see below), are consistent with an axonal pathway from the retina to the LoC via axo-axonic synapses in the tectum.

\section{Evidence for an additional, systemic route for NGF from retina to the LoC}

To determine whether the axo-axonic pathway was the only route responsible for accumulation of eye-derived NGF in the LoC, we applied one of two axonal transport inhibitors to the eye (von Bartheld et al., 1996a,b): colchicine that abolishes all axonal transport, most likely due to depolymerization of microtubules, or monensin that eliminates anterograde axonal transport, presumably due to disruption of the uptake and processing of proteins for anterograde transport at the level of the Golgi system (Hammerschlag et al., 1982), but leaves retrograde transport intact. The tectum served as a positive control for monensin coinjection, and both the tectum and ION served as positive controls for colchicine coinjection. Colchicine completely abolished both retrograde and anterograde axonal transport from the eye to the tectum and ION (Fig. 2 E), and monensin nearly eliminated anterograde transport with no effect on retrograde transport to the ION (Fig. 2E). The LoC, however, continued to accumulate eye-derived NGF in both conditions, although this accumulation was reduced by $\sim 40 \%$ with monensin and by $\sim 50 \%$ with colchicine (Fig. $2 E$ ). These reductions were statistically significant (colchicine vs NGF only, $p=0.025$; monensin vs NGF only, $p=0.027$; one-way ANOVA). Thus, our data show that the axonal route contributes to only $40-50 \%$ of the total NGF accumulation in the LoC, and that the LoC can accumulate NGF by an additional, nonaxonal route. This interpretation is supported by two additional observations: NGF reached the LoC as early as $4 \mathrm{~h}$ after intraocular injection (which coincides with the earliest appearance of NGF at $4-5 \mathrm{~h}$ in the tectum and ION) (Butowt and von Bartheld, 2009), and NGF accumulated in the LoC bilaterally and almost symmetrically, despite the nearly exclusive unilateral trafficking of NGF to the tectum and ION. In the LoC, the majority $(\sim 60-65 \%)$ of noradrenergic neurons were labeled above background, when compared with sections labeled with dopamine- $\beta$ hydroxylase antibodies (von Bartheld and Bothwell, 1992). Together, these data establish a nonaxonal route as a major pathway of NGF from the eye to the LoC.

NGF transcytoses through ependymal tanycytes to reach the LoC after injection in the CSF

No NGF accumulated in the LoC after injection into the vascular circulation (data not shown), but when radiolabeled NGF was 
injected directly into the cerebral ventricles, it accumulated bilaterally and specifically in the LoC (Fig. 3A). NGF distributed in a pattern that was indistinguishable from the one seen after intraocular injection, except for a stronger signal. NGF also accumulated in a region immediately adjacent to the ependyma, exclusively at the level of the LoC (Fig. 3A). This accumulation in and adjacent to the LoC was specific for NGF, as it was not seen with other radiolabeled proteins such as GDNF (Fig. 3B). Although LoC neurons have never been reported to possess neurites that penetrate the ependyma and directly contact the CSF of the fourth ventricle, unlike some CSF-contacting neurons in the third ventricle (von Bartheld and Meyer, 1990; Vigh and Vigh-Teichmann, 1998; Nieuwenhuys, 2000), it is at least theoretically possible that LoC neurons directly internalize NGF from the CSF. Alternatively, NGF may move by transcytosis through an intermediary ependymal cell, the tanycyte (Rodriguez et al., 2005), which contacts CSF as well as LoC neurons (Felten et al., 1981b). To distinguish between these two possibilities, we examined $>350,000$ $\mu \mathrm{m}^{2}$ of ultrathin sections through the ependyma adjacent to the LoC without evidence for even a single LoC (or other) neuronal process penetrating the ependyma. In addition, we traced the fate of radiolabeled NGF after intraventricular injection by quantitative ultrastructural autoradiography. These experiments confirmed that NGF accumulated in LoC neuronal cell bodies (Fig. 3C), but no labeled (or unlabeled) processes of LoC neurons, or of any other cells, were found that penetrated the ependymal layer. Instead, a distinct type of tanycyte, the $\beta$-tanycyte, was heavily labeled (Fig. $3 D)$. Tanycytes in the third ventricle have been well studied and documented (Rodriguez et al., 2005), but very little is known about those in the fourth ventricle. Nevertheless, based on the information available on third ventricle tanycytes, the tanycytes differ substantially in electron density and nuclear shape and size from normal ependymal cells (Fig. 3E). Furthermore, different subtypes of tanycytes can easily be distinguished in the ependyma adjacent to the LoC in the fourth ventricle (supplemental Fig. $1 A, B$, available at www.jneurosci.org as supplemental material). Examination of the neuropil indicated that radiolabeled NGF moved along tanycyte processes into the neuropil (Fig. 4A) and then accumulated in axonal terminals that contained large dense-cored vesicles and formed synaptoid contacts with tanycyte "shafts" (Guldner and Wolff, 1973; Scott et al., 1974; Rodriguez et al., 2005) (Fig. 4B). Silver grains in tanycytes did not accumulate in lysosomes (data not shown). Quantification of labeling densities over cell types and compartments (Fig. $4 C, D$ ) indicated transcytosis through tanycytes (types $\beta 1$ and $\beta 2$ ) (supplemental Fig. $1 B, C$, available at www.jneurosci.org as supplemental material) (Rodriguez et al., 2005), but not through $\alpha$-tanycytes or regular ependymal cells (Fig. 3E). Transfer of NGF occurred via microtubule-loaded tanycyte processes that contact axonal terminals (so-called synaptoid contacts) (Guldner and Wolff, 1973), extending from adjacent LoC neurons. Since the processes of different tanycyte subtypes cannot be distinguished for calculation of fractional area and labeling densities, the labeling den- sity of $\beta$-tanycytes, and especially $\beta 2$-tanycytes, may be underestimated in Figure $4 D$. Together, these data indicate that LoC neurons do not directly contact the CSF, but that NGF is transcytosed through $\beta 2$-tanycytes to eventually accumulate in LoC neurons, as illustrated schematically (Fig. 5).

\section{Specificity of NGF accumulation and NGF receptor expression}

Besides NGF in birds, NT-3 and GDNF have been implicated in mammalian LoC trophism (Arenas et al., 1995). When these and other radiolabeled proteins were injected into the CSF, none of the other proteins accumulated in the LoC [GDNF (Fig. 3B); NT-3, FGF2, IGF1 (data not shown)]. The specificity of accumulation of CSF-derived NGF in the LoC suggested that this process is receptor mediated and that the CSF-contacting tanycytes may express NGF receptors (p75 and/or trkA). Consistent with this hypothesis, immunocytochemical studies at the light- and electron-microscopic levels showed specific labeling for both trkA and 75 receptors in presumptive tanycytes, including labeling with an antibody against the cytoplasmic domain of p75 (Fig. $6 A, B, F, G)$. The processes immediately beneath the ependyma also showed specific labeling for trkA, p75, and tyrosine hydroxylase (Fig. 6C-E). Our in situ hybridization studies, however, did not reveal any labeled cells in the ependymal layer, despite robust expression of p75 and trkA receptors in the adjacent LoC neurons (Fig. $6 H, I)$. These data are consistent with previous reports of barely detectable p75 mRNA expression in ependymal tanycytes of the third ventricle despite strong immunolabeling of the protein (Gibbs et al., 1989; Koh et al., 1989; Rodriguez et al., 2005). They support the conclusion that tanycytes express NGF receptor mRNA at low levels, below detection by in situ hybridization. The discrepancy between in situ hybridization and immunocyto- 

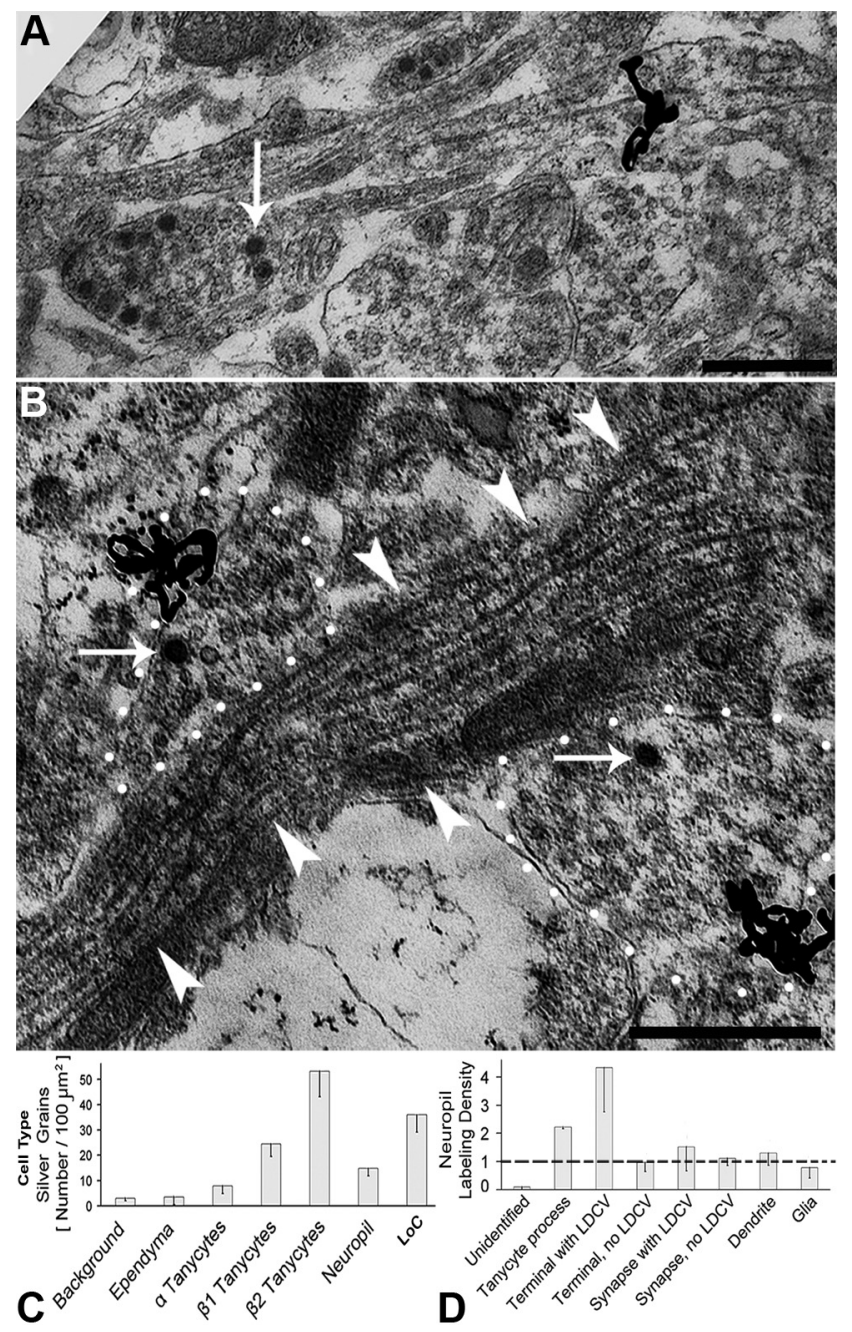

Figure 4. The route of NGF from the CSF to the LoC: quantitative ultrastructural analysis implicates tanycytes. $\boldsymbol{A}, \boldsymbol{B}$, After injection of radiolabeled NGF into the ventricle, silver grains accumulated at the ultrastructural level within tanycyte processes $(\boldsymbol{A})$ and over synaptoid contacts ( $\boldsymbol{B}$, outlined by white dots) adjacent to tanycyte processes (arrowheads). Presumptive LoC terminals lack synaptic densities but form synaptoid contacts that contain large dense-cored vesicles (LDCVs; white arrows). Synaptoid contacts preferentially associate with tanycyte processes. C, Quantification of radiolabeled NGF over identified cell types located in regions from the ependyma to the LoC. NGF accumulated exclusively in $\beta 1$ - and $\beta 2$-tanycytes and LoC neurons. $n=3-6 . D$, Quantification and comparison of silver grain labeling densities of identified structures within the neuropil, including terminals, dendrites, glia, and tanycyte processes. Note high labeling densities for tanycyte processes and terminals containing LDCVs, but only background (labeling density, 1; dashed line) for all other components of this neuropil. Error bars indicate SEM. Number of independent experiments, $n=3-6$. Scale bars: $A, B, 500 \mathrm{~nm}$.

chemistry data is most likely due to low turnover and slow degradation of the receptor protein that is used for shuttling rather than signaling. This is consistent with the apparent lack of NGF in lysosomes of tanycytes and the apparent lack of degradation of CSF-derived dopamine in third ventricle tanycytes (Scott et al., 1974), in contrast to NGF accumulation in neuronal lysosomes when NGF signals within cells (Claude et al., 1982).

Comparison of efficiencies of axonal and systemic routes of NGF accumulation in the LoC

To directly compare the efficiencies of eye-derived NGF accumulation for the axonal and systemic routes, we measured concentrations of exogenous NGF in both the retinal target and the CSF,

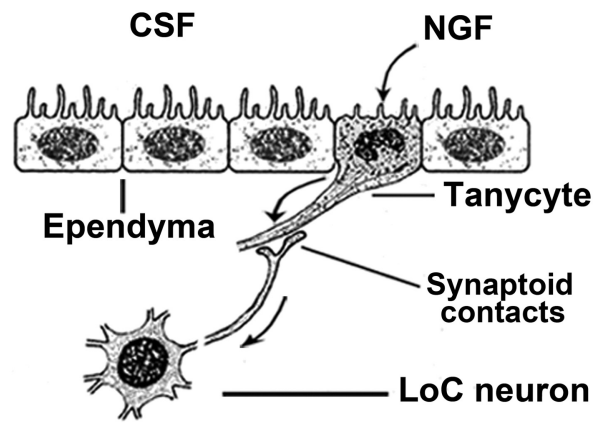

Figure 5. Synopsis of NGF extraction from CSF via tanycytes and accumulation in LoC neurons. Schematic summary of spatial relationships between cell types from the $\mathrm{LoC}$ neurons to the CSF, including tanycytes. NGF trafficking pathways (arrows) between the CSF and the LoC neurons via tanycytes are based on silver grain accumulations shown in Figure 4.

and the relative amount of NGF that accumulated in the LoC by each route (Table 1). Based on gamma counts of dissected tissues and/or fluid samples, after intraocular injection of 50-100 ng of radiolabeled NGF, the optic tectum accumulated $\sim 115 \mathrm{pg}$, and the CSF contained $\sim 8 \mathrm{pg}$, of NGF. The LoC contained $3 \mathrm{pg}$, with approximately half of that $(1.5 \mathrm{pg})$ via the axonal route, and another $1.5 \mathrm{pg}$ retrieved from the CSF, since approximately half of the radioactivity in the LoC could be abolished by axonal transport inhibitors (Fig. 2E; Table 1). The amount of $1.5 \mathrm{pg}$ transported retrogradely along axons by $\sim 600-700$ LoC neurons is similar to previous estimates that 14,000 isthmo-optic neurons transport a maximum of $\sim 20 \mathrm{pg}$ of radiolabeled neurotrophins within $20 \mathrm{~h}[\sim 33,000$ molecules per isthmic neuron (von Bartheld et al., 1996b); 50,000 molecules per LoC neuron]. A major fraction of radiolabeled NGF in the CSF could be precipitated by trichloroacetic acid, and this fraction was stable in the CSF over time (data not shown). Therefore, trafficked NGF likely constituted intact NGF rather than free iodine or minor degradation products. LoC neurons extract $\sim 1.5 \mathrm{pg}$ of NGF from $115 \mathrm{pg}$ of NGF in the optic tectum, but the same amount from the $8 \mathrm{pg}$ of NGF in the CSF. Thus, the uptake of NGF via ependymal tanycyte transcytosis is $\sim 15$-fold more efficient than the transport (via RGC and LoC axons) across axo-axonic synapses in the optic tectum. Our data show that the tanycyte-associated LoC can use CSF-derived neurotrophic factors as a source of trophic supply, as was suggested decades ago (Felten et al., 1981b). We conclude that neuronal populations that use the CSF-tanycyte extraction route can efficiently obtain trophic factors through the CSF.

Specificity of the tanycyte transcytosis route for LoC neurons Noradrenergic, p75/trkA-expressing LoC neurons overlap with another population, the FGF-receptor-expressing cholinergic neurons in the avian brainstem (von Bartheld and Bothwell, 1992). Since occasional tanycytes were observed in the fourth ventricular ependyma without any trkA or p75 immunolabel (data not shown), we examined whether those tanycytes may functionally associate with the cholinergic, FGF receptorexpressing neurons below the fourth ventricle or whether only the noradrenergic LoC neurons have such privileged access to the CSF. When we injected radiolabeled FGF2 in the CSF of E16 chick embryos, we observed no specific uptake beyond the immediate diffusion area adjacent to the ependyma (Fig. $6 L$ ). Thus, our data indicate that these cholinergic neurons lack a functional tanycyte association. We also tested the distribution of other radiolabeled growth factors after intraventricular injection. No accumulation in the LoC was found [GDNF (Fig. 3B); NT-3, IGF1, FGF2 (data 
not shown)]. This further emphasizes the specificity of the tanycyte-mediated interface between the noradrenergic LoC neurons and the CSF for transfer of signaling molecules from the CSF. Tanycytes lacking NGF receptors may associate with serotonergic raphe brainstem neurons as suggested for the fourth ventricle in rabbits (Felten et al., 1981a,b).

\section{Exogenous NGF in the CSF increases} the nuclear diameters of LoC neurons To determine whether NGF in the CSF has significant physiological effects on LoC neurons, we conducted a series of gain-of-function experiments. We chose karyometric studies of LoC neurons as a readout for assessment of LoC function (Bubenik and Monnier, 1972). It is well established that LoC neurons rapidly and reliably change their nuclear sizes with their level of activity; i.e., during sleep the nuclear size decreases, whereas arousal, stress, or treatment with antidepressive medication increases nuclear size (Bubenik and Monnier, 1972; Smialowska et al., 1988). These effects have been directly correlated with electrical activity (firing rates) of LoC neurons, as well as changes in the activity levels of tyrosine hydroxylase (Olpe and Jones, 1983; Racagni and Brunello, 1984). When small amounts (50-100 ng) of exogenous NGF were introduced into the ventricular system of chick embryos, the LoC neurons (identified by tyrosine hydroxylase immunolabeling) increased their nuclear size significantly, compared with controls (Fig. 7E,F). Importantly, this effect was seen only in the LoC proper (but not in the nucleus subceruleus that also expresses trkA) (Fig. 7I), but does not have access to the CSF via tanycytes and is never labeled through the tanycyte uptake route, neither after direct injection of radiolabeled NGF in the CSF nor after intraocular injection (Fig. 7H). These data show that CSF-derived NGF can affect physiological parameters of LoC neurons, apparently via tanycyte transcytosis.

\section{Evidence that endogenous NGF in the CSF maintains LoC nuclear sizes}

Since the eye is believed to be one of many sources of endogenous NGF in the CSF, exclusively eliminating this source may result in minimal reduction of NGF in the CSF. Therefore, we did not attempt to eliminate retinal or eye-derived NGF, but rather chose to directly manipulate the levels of endogenous NGF in the CSF (loss-of-function experiments) to determine whether endogenous NGF in the CSF has effects on LoC nuclear size (and thus LoC neuronal activities) (Bubenik and Monnier, 1972; Olpe and Jones, 1983; Racagni and Brunello, 1984). We obtained a neutralizing antibody against NGF and confirmed that it recog$H, L, 200 \mu \mathrm{m} ; F, G, 4 \mu \mathrm{m}$.
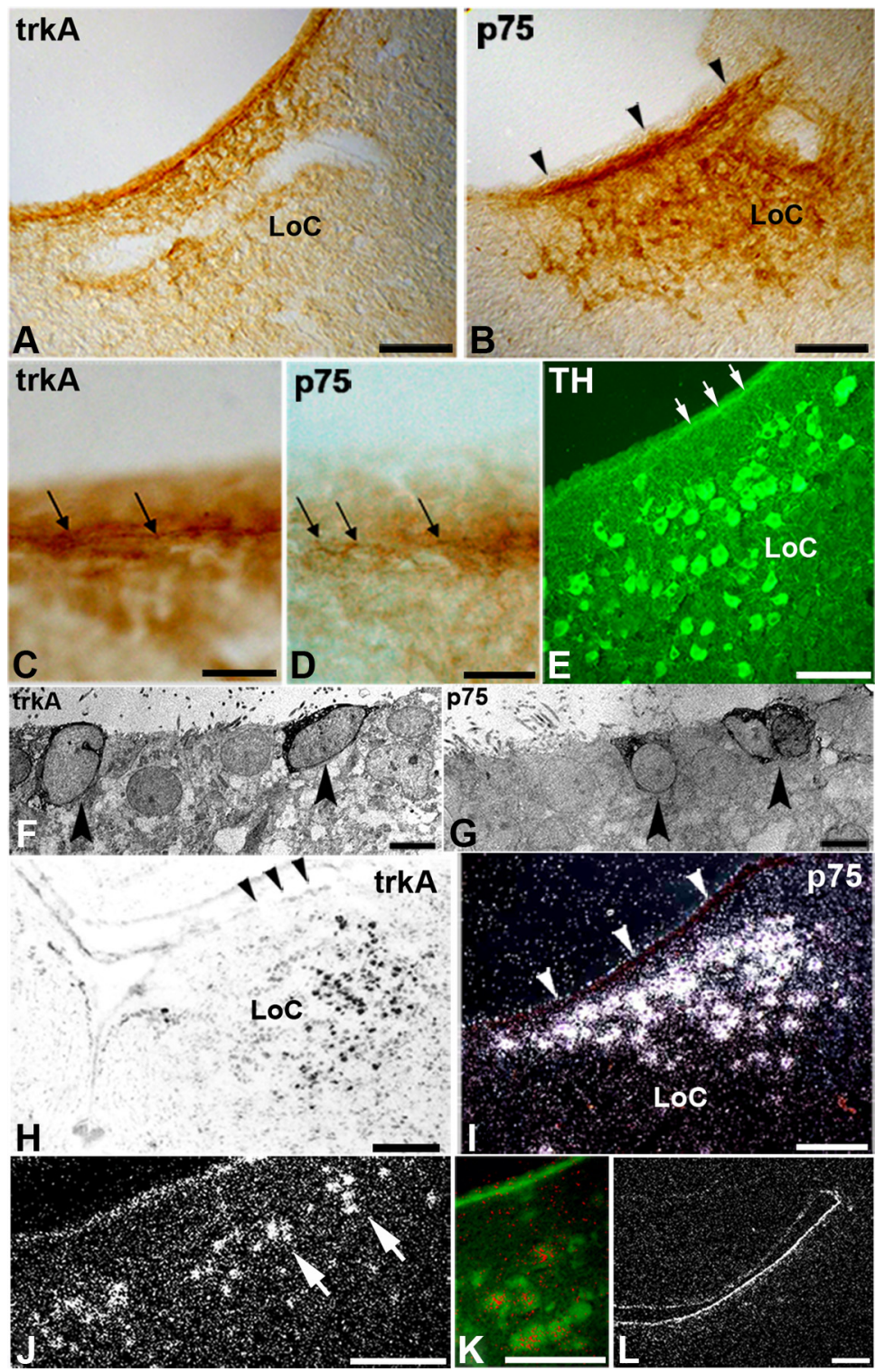

Figure 6. Localization of NGF receptors in the $L 0 C$ and accumulation of the stress hormone urotensin-1. $A, B, L$ LC neurons and the ependyma adjacent to the $L_{0}($ were immunolabeled with antibodies against trkA $(\boldsymbol{A})$ and p75 receptors ( $\boldsymbol{B}$, arrowheads). $\boldsymbol{C}, \boldsymbol{D}$, Immunolabels for trkA and p75 protein were located in or immediately adjacent to the ependymal layer (arrows) exclusively at the level of the LoC. $E$, The ependyma adjacent to the LoC contained tyrosine-hydroxylase-immunolabeled nerve fibers (arrows) in the same position as the labeled trkA and 75 (compare with $\mathbf{C}, \mathbf{D})$. $\boldsymbol{F}, \mathbf{G}$, At the ultrastructural level, tanycytes (arrowheads) were labeled for both trkA $(\boldsymbol{F})$ and p75 $(\boldsymbol{G})$, using an antibody that recognizes exclusively the intracellular domain of $\mathrm{p} 75$. This suggests that tanycytes express $\mathrm{p} 75$ rather than obtaining a cleaved extracellular $\mathrm{p} 75$ protein fragment. $\boldsymbol{H}, \boldsymbol{I}, \mathbf{I n}$ situ hybridization at E13-E18 shows that LoC neurons heavily express trkA ( $\boldsymbol{H}$, bright-field image) and p 75 mRNA (I, dark-field image), but no cell bodies in the adjacent ependymal layer (arrowheads) were labeled above the level of detection. $\boldsymbol{J}, \boldsymbol{K}$, Radiolabeled urotensin- 1 accumulated in a similar fashion as NGF in the LoC neurons (arrows) after intraventricular injection. Double labeling with aldehyde catecholamine fluorescence (green) for catecholamines and radiolabel (converted to red for easier visualization) for urotensin-1 show that urotensin-1 accumulated in LoC neurons. $L$, Specificity of urotensin-1 accumulation is demonstrated by lack of accumulation of radiolabeled FGF2 in the brain parenchyma after similar intraventricular injection. Scale bars: $A, B, E, I, J, K, 100 \mu \mathrm{m} ; \boldsymbol{C}, \boldsymbol{D}, 10 \mu \mathrm{m}$;

nizes chicken NGF (Fig. $7 B-D$ ). This antibody is highly selective for NGF, with minimal cross-reactivity to other neurotrophins (Fig. 7A). When this antibody was injected into the ventricular system of chick embryos, the LoC neurons selectively decreased their nuclear diameter within $48 \mathrm{~h}$, apparently due to elimination of the trophic effects of endogenous NGF on LoC neurons. There was no such effect on the nuclear size of the neurons in the nucleus subceruleus (Fig. 7I,J), and no LoC-specific effect was seen 
Table 1. Tracking of radiolabeled NGF from the eye to the LoC in 18-d-old chick embryos by gamma counting

\begin{tabular}{|c|c|c|c|c|c|c|}
\hline \multirow[b]{2}{*}{ Structure } & \multirow[b]{2}{*}{ Cell type } & \multirow[b]{2}{*}{ Number } & \multirow{2}{*}{$\begin{array}{l}\text { Volume } \\
\text { (in } \mu \mathrm{l} \text { ) }\end{array}$} & \multicolumn{2}{|l|}{$\left.\right|^{125}$-NGF } & \multirow{2}{*}{$\begin{array}{l}\text { Molecules } \\
\text { per cell or } \\
\text { axon }\end{array}$} \\
\hline & & & & (in cpm) & (in pg) & \\
\hline Eye & RGCS & $3 \times 10^{6}$ & & $4.84 \times 10^{6}( \pm 0.36)$ & $60,563( \pm 4467)$ & \\
\hline Tectum & $\mathrm{RGC}_{\text {axons }}{ }^{a}$ & $3 \times 10^{6}$ & & $9236( \pm 1927)$ & $115( \pm 24.1)$ & 881.7 \\
\hline LoC & Neurons & $600-700^{b}$ & & $243( \pm 29.3)$ & $3.03( \pm 0.36)$ & 107,215 \\
\hline CSF & & & $52( \pm 6.4)$ & $642.7( \pm 50.6)$ & $8.03( \pm 0.63)$ & \\
\hline
\end{tabular}

Numbers in brackets indicate SEM. Molecules are neurotrophin dimer molecules. RGC, retinal ganglion cell. The counts per minute and picograms for CSF are calculated after correction for precipitated counts.

${ }^{a}$ Axon numbers according to Butowt and von Bartheld (2001).

${ }^{b}$ Neuron numbers according to von Bartheld and Bothwell (1992). The amount of neurotrophin transported retrogradely along axons on a per-cell basis is similar to that reported previously (von Bartheld et al., 1996b).

when control IgG was injected into the ventricle or when PBS was used. Therefore, we conclude that endogenous NGF within the CSF contributes to the control of nuclear size and thus the activity level of LoC neurons.

\section{Time course study of karyometric effects of NGF levels in the CSF}

To determine how rapidly NGF levels in the CSF may affect measurable changes in nuclear sizes of LoC neurons (and thus, by analogy, changes in the physiological activity of these neurons) (Bubenik and Monnier, 1972; Olpe and Jones, 1983; Racagni and Brunello, 1984), we examined effects of reduced or enhanced NGF levels in the CSF at 4, 24, and 48 h. As shown in Figure 7J, there was no significant effect in either gain- or loss-of-function experiments at $4 \mathrm{~h}$, although single injections of NGF or NGF antibody caused significantly increased (+NGF) or decreased $(-\mathrm{NGF})$ nuclear sizes at $24 \mathrm{~h}$. The magnitude of the changes was less than that observed after $48 \mathrm{~h}$. We conclude that NGF levels in the CSF regulate morphological and, presumably, physiological properties of LoC neurons within $24 \mathrm{~h}$ or less. This time frame is intermediate between previous reports that indicated changes in nuclear sizes of adult mammalian LoC neurons within $<1 \mathrm{~h}$ (Bubenik and Monnier, 1972) or in the order of weeks of manipulations (Smialowska et al., 1988).

\section{A CRF family member, urotensin-1, also accumulates in LoC neurons}

Locally or intraventricularly applied CRF family members are highly efficient in activating LoC neurons (Valentino and Van Bockstaele, 2008). To determine whether the tanycyte transcytosis route from the CSF to the LoC is restricted to NGF, we tested a CRF family member, urotensin-1. Urotensin-1 was chosen because it was shown previously in avian species to have a stronger binding affinity to the CRF receptor 1 than CRF (Yu et al., 1996). After intraventricular injection, radiolabeled urotensin-1 readily accumulated in the LoC in a pattern that was similar to that of radiolabeled NGF (compare Figs. $3 A, 6 J$ ). Double labeling with formaldehyde-induced catecholamine fluorescence verified the identity of the LoC neurons (Fig. $6 \mathrm{~K}$ ). The specificity of urotensin- 1 accumulation was shown by the absence of accumulation of FGF2 (Fig. 6L). Thus, stress-related hormones of the CRF family can be extracted from the CSF and transferred to LoC neurons in a pattern that appears identical to that shown for radiolabeled NGF.

\section{Discussion}

In the context of neuronal signaling within the brain, "wiring transmission" (via axonal transport and synaptic contacts) is considered the primary mode and far more important than "vol- ume transmission" (via extrasynaptic diffusion) (Bennett, 1999; Bloom, 2000). Our work shows that for NGF and possibly for CRF family members, volume transmission from the CSF can contribute a surprisingly large fraction of the signaling molecules that accumulate in the LoC. By comparing the contributions of two different routes, an axonal pathway and a systemic pathway, we provide evidence for the physiological significance of a largely neglected pathway-CSF circulation and transcytosis through a specialized ependymal cell type, the tanycyte. These findings have implications for both the trafficking of endogenous trophic factors as well as the delivery of exogenous trophic factors for therapeutic purposes.

New insights into the axonal pathway of signaling molecules Previous work on the trafficking of signaling molecules has focused on dendrite-to-axon transfer (classical neurotrophic hypothesis) (Purves, 1988) as well as axodendritic transfer (von Bartheld et al., 1996b; Kohara et al., 2001) and glia-to-neuron transfer (Du and Dreyfus, 2002). Axo-axonic transfer of a neurotrophic factor has, to our knowledge, not been reported previously. In the case of the retina-LoC pathway, axo-axonic transfer of the ligand involves receptor switching, from p75 to trkA. Apparently, NGF is released from p75 on RGC axon terminals after anterograde transport and subsequently binds to trkA (or a trkA/ p75 complex) for internalization into the presynaptic ceruleus axon terminal and retrograde transport to LoC cell bodies. Together, these findings support the concept that neurotrophic factors can be recycled and are not necessarily degraded upon internalization (von Bartheld et al., 2001).

\section{Spillover of peptides from the eye to the CSF}

Multiple sources are believed to supply growth factors into the CSF, e.g., CSF-contacting neurons (Vigh and Vigh-Teichmann, 1998), choroid plexus (Mashayekhi et al., 2009), and astrocytes and oligodendrocytes (Hochhaus et al., 2001). In the case of NGF, the combination of two findings, exceptionally high expression of NGF in the eye (Large et al., 1989; Lambiase et al., 2002) and increasing evidence for penetration of small amounts of eyederived proteins such as insulin and NGF into the CSF (Koevary et al., 2003, 2004; Lambiase et al., 2007), suggest that eye-derived NGF may contribute to the endogenous NGF that is known to be present in the CSF of all vertebrates examined (Xia et al., 2000; Hochhaus et al., 2001; Chiaretti et al., 2008, 2009; Mashayekhi et al., 2009). Our study shows that exogenous NGF, when applied intraocularly, gains access to intraventricular CSF spaces. Since the optic nerve and the optic nerve head are bathed in CSF-filled subarachnoid space (Shen et al., 1985; Ludemann et al., 2005; Killer et al., 2006), it is not surprising that physiologically relevant amounts of eye-derived NGF reach the systemic CSF circulation and gain entry into ventricular spaces, from which they can reach "upstream" ventricular CSF spaces (Dickson et al., 2007).

\section{Evidence for a novel systemic pathway through the CSF}

The most significant finding of our study is the evidence that the systemic CSF pathway can be a major contributor to the accumulation of functionally relevant signaling molecules in the LoC. In the past, the CSF has been largely neglected as a pathway of endogenous neurotrophic factors (Nicholson, 1999). The finding of an efficient and molecule-specific volume transmission pathway from the CSF to the LoC is of considerable interest, given the LoC's clinical and pharmacological importance (Berridge and Waterhouse, 2003). The newly discovered CSF-tanycyte-LoC signaling pathway will allow us to better understand LoC func- 
tion and may provide a novel approach to exploit this pathway for therapeutic purposes.

\section{Mechanisms of tanycyte transcytosis}

Our work suggests that tanycytes control the information flow from the CSF to the LoC (and possibly in the reverse direction as well) in a way that resembles, but is not identical to, the function of tanycytes in the control of hypothalamic neurosecretion (Bruni, 1998; Rodriguez et al., 2005). Whereas tanycytes in the third ventricle have been the subject of numerous studies (Rodriguez et al., 2005), the function of tanycytes in the ependyma of the fourth ventricle is enigmatic. We now show that these tanycytes provide a specific and functionally relevant interface between the LoC and the CSF in the fourth ventricle. This structural arrangement and role are similar to that of tanycytes in the third ventricle, but differ in that the hypothalamic tanycytes interface between neuroendocrine hypothalamic neurons and the portal blood system, controlling neurosecretion into the blood (Rodriguez et al., 2005). The fourth ventricle tanycytes, however, interface between CSF spaces and the LoC neurons, and control the uptake and transfer of signaling molecules from the CSF to the LoC. The extraction of signaling molecules from the CSF exhibits remarkable specificity; the tanycyte transcytosis is highly selective for distinct trophic factors: NGF, but not GDNF, NT-3, or FGF2. This indicates that tanycytes and/or LoC neurons are actively screening for specific factors rather than internalizing them by diffusion or bulk transport. Furthermore, the LoC seems unique or among a small number of neuronal populations with such privileged access to the CSF, as this does not apply to the cholinergic, FGFreceptor-expressing tegmental neurons in close vicinity. The mechanism of NGF accumulation in the LoC involves highly efficient extraction from the CSF. When compared with the multisynaptic axonal trafficking pathway from the eye, CSF extraction is significantly more efficient (Table 1), consistent with our finding that this volume transmission pathway has a major influence on LoC function.

\section{Evidence for functional significance of NGF in the CSF}

Previous studies have shown that NGF controls the physiological activity of trkA-expressing neurons (Levi-Montalcini et al., 1975; Hendry, 1976; Hendry and Campbell, 1976; Hagg et al., 1989; von Bartheld et al., 1995). NGF in the CSF affects neuronal proliferation in embryos (Mashayekhi and Salehi, 2007), amygdaloid kindling in mature animals (Funabashi et al., 1988; Van der Zee et al., 1995), and LoC function (determined by karyometric analysis) (present study). No previous studies have examined the role
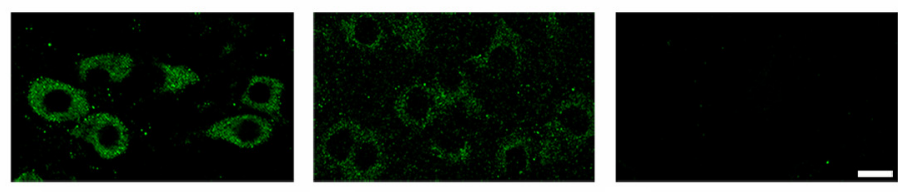

C LoC

D Negative Control

B Trigeminal Nucleus
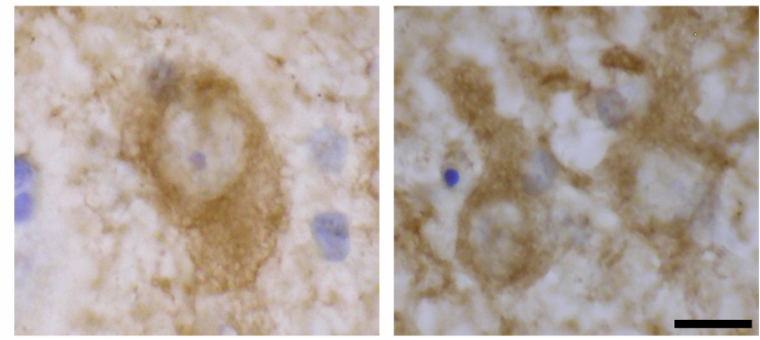

F $\quad$ LoC (control)

G

LoC (-NGF)
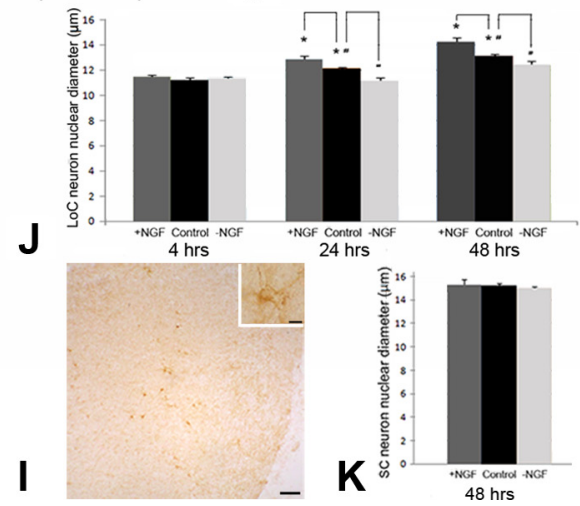

Figure 7. Both endogenous and exogenous NGF in the CSF regulate the nuclear sizes of LoC neurons. $\boldsymbol{A}$, Dot blot experiments show that our polyclonal neutralizing NGF antibody specifically recognizes NGF with minimal cross-reactivity for BDNF and NT-3. nal motor neurons showed no labeling when the primary antibody was omitted (D). $\boldsymbol{E}-\boldsymbol{G}$, Evidence that both exogenous and (SC; outlined by green dots). $I$, The subceruleus neurons contain both p75 and trkA receptors (trkA immunolabel shown), PBS (control), or neutralizing endogenous NGF in the CSF (-NGF). Number of independent experiments: + NGF, - NGF, neurons, demonstrating the specificity of the NGF effect on LoC. Error bars indicate SEM. Scale bars: $\boldsymbol{D}$ (for $\boldsymbol{B}-\boldsymbol{D}), \boldsymbol{G}($ for $\boldsymbol{E}-\boldsymbol{G}), 10$ $\mu \mathrm{m} ; \boldsymbol{H}, 200 \mu \mathrm{m} ; \boldsymbol{I}, 100 \mu \mathrm{m}$; inset, $10 \mu \mathrm{m}$.

of either exogenous or endogenous NGF in the CSF on the main producers of noradrenaline in the CNS, the LoC, and the possible route of how CSF-derived growth factors and hormones may influence this major CNS population. By using a karyometric and quantitative autoradiographic approach, we demonstrate not only that CSF-derived NGF controls avian LoC nuclear size, known to be linked to neuronal activity of the LoC (Bubenik and Monnier, 1972; Smialowska et al., 1988), but also that LoC neurons gain access to the NGF that is present within the CSF by using a novel mechanism: tanycyte-mediated extraction and transcytotic transfer of NGF. Importantly, while responses of the LoC to NGF are bird specific, the tanycyte-LoC connection is also found in adult mammals (Felten et al., 1981b). However, this relationship was not examined previously at the ultrastructural or functional level. 


\section{The CSF-tanycyte-LoC pathway: a one-way or two-way street?}

An important question for the future is whether the tanycyte interface is designed merely for unidirectional import of CSFderived signaling molecules such as neuropeptides and trophic factors (Bjelke and Fuxe, 1993; Fernandez-Galaz et al., 1996), or whether it also facilitates and regulates bidirectional transport and release of signaling molecules from the LoC to the CSF. Noradrenaline in the CSF is associated with multiple diseases (Hyland, 2008). It is currently assumed that noradrenaline diffuses through brain parenchyma in a "random fashion" from myriad neuropil release sites and gains access to the CSF as a "sink" (Nicholson, 1999). Based on our new insights into tanycyte structure and function and their spatial relationship with LoC neurons, we suggest an alternative model: release of noradrenaline into the CSF may be specifically regulated and controlled via the tanycyte compartment, similar to the release of gonadotropinreleasing hormone from hypothalamic neurons via tanycytes into the portal venous blood stream (Rodriguez et al., 2005).

Intraventricular application of exogenous trophic factors can have therapeutic effects in neurodegenerative diseases (Mufson et al., 1999). In this context, it is of interest that in patients with Alzheimer's disease, LoC neurons degenerate at an even faster rate than cholinergic basal forebrain neurons (Zarow et al., 2003). Given the highly efficient extraction rate via tanycytes, even moderately elevated levels of relevant trophic factors in the CSF may suffice to support those neuronal populations, such as the LoC, that have a highly efficient "back-door" interface and uptake system for trophic factors from the CSF. Better understanding of the molecular mechanisms underlying the remarkable efficiency of CSF extraction could provide new avenues for therapeutic drug delivery to the LoC, which constitutes one of the most important pharmaceutical targets in the CNS, since the LoC controls experience-dependent alterations in behavior (Berridge and Waterhouse, 2003). A better understanding of the specificity of the tanycyte transcytosis route and the pathways between the eye and the CSF spaces may allow the development of eye-topical application (Di Fausto et al., 2007; Lambiase et al., 2007) to target delivery of drugs to one of the clinically most important CNS centers, and potentially with minimal side effects.

\section{References}

Agnati LF, Zoli M, Stromberg I, Fuxe K (1995) Intercellular communication in the brain: wiring versus volume transmission. Neuroscience 69:711-726.

Angaut P, Reperant J (1976) Fine structure of the optic fibre termination layers in the pigeon optic tectum: a Golgi and electron microscope study. Neuroscience 1:93-105.

Arce EA, Bennett-Clarke CA, Rhoades RW (1994) Ultrastructural organization of the noradrenergic innervation of the superficial gray layer of the hamster's superior colliculus. Synapse 18:46-54.

Arenas E, Trupp M, Akerud P, Ibanez CF (1995) GDNF prevents degeneration and promotes the phenotype of brain noradrenergic neurons in vivo. Neuron 15:1465-1473.

Bennett MR (1999) The early history of the synapse: from Plato to Sherrington. Brain Res Bull 50:95-118.

Berridge CW, Waterhouse BD (2003) The locus coeruleus-noradrenergic system: modulation of behavioral state and state-dependent cognitive processes. Brain Res Brain Res Rev 42:33-84.

Bjelke B, Fuxe K (1993) Intraventricular beta-endorphin accumulates in DARPP-32 immunoreactive tanycytes. Neuroreport 5:265-268.

Bloom FE (2000) Integration of wiring transmission and volume transmission. Prog Brain Res 125:21-26.

Bruni JE (1998) Ependymal development, proliferation, and functions: a review. Microsc Res Tech 41:2-13.
Bubenik G, Monnier M (1972) Nuclear size variations in cells of the locus ceruleus during sleep, arousal and stress. Exp Neurol 35:1-12.

Butowt R, von Bartheld CS (2001) Sorting of internalized neurotrophins into an endocytic transcytosis pathway via the Golgi system: ultrastructural analysis in retinal ganglion cells. J Neurosci 21:8915-8930.

Butowt R, von Bartheld CS (2009) Fates of neurotrophins after retrograde axonal transport: phosphorylation of p75NTR is a sorting signal for delayed degradation. J Neurosci 29:10715-10729.

Chiaretti A, Antonelli A, Genovese O, Pezzotti P, Rocco CD, Viola L, Riccardi R (2008) Nerve growth factor and doublecortin expression correlates with improved outcome in children with severe traumatic brain injury. J Trauma 65:80-85.

Chiaretti A, Barone G, Riccardi R, Antonelli A, Pezzotti P, Genovese O, Tortorolo L, Conti G (2009) NGF, DCX, and NSE upregulation correlates with severity and outcome of head trauma in children. Neurology 72:609-616.

Claude P, Hawrot E, Dunis DA, Campenot RB (1982) Binding, internalization, and retrograde transport of 125I-nerve growth factor in cultured rat sympathetic neurons. J Neurosci 2:431-442.

Del Bigio MR (1995) The ependyma: a protective barrier between brain and cerebrospinal fluid. Glia 14:1-13.

Dickson P, McEntee M, Vogler C, Le S, Levy B, Peinovich M, Hanson S, Passage M, Kakkis E (2007) Intrathecal enzyme replacement therapy: successful treatment of brain disease via the cerebrospinal fluid. Mol Genet Metab 91:61-68.

Di Fausto V, Fiore M, Tirassa P, Lambiase A, Aloe L (2007) Eye drop NGF administration promotes the recovery of chemically injured cholinergic neurons of adult mouse forebrain. Eur J Neurosci 26:2473-2480.

Du Y, Dreyfus CF (2002) Oligodendrocytes as providers of growth factors. J Neurosci Res 68:647-654.

Felten DL, Cummings JP, Burnett BT (1981a) Ontogeny of caudal fourth ventricular tanycytes in the rabbit brain: a Golgi study. Anat Rec 200:321-330.

Felten DL, Harrigan P, Burnett BT, Cummings JP (1981b) Fourth ventricular tanycytes: a possible relationship with monoaminergic nuclei. Brain Res Bull 6:427-436.

Fernandez-Galaz MC, Torres-Aleman I, Garcia-Segura LM (1996) Endocrine-dependent accumulation of IGF-I by hypothalamic glia. Neuroreport 8:373-377.

Funabashi T, Sasaki H, Kimura F (1988) Intraventricular injection of antiserum to nerve growth factor delays the development of amygdaloid kindling. Brain Res 458:132-136.

Gibbs RB, McCabe JT, Buck CR, Chao MV, Pfaff DW (1989) Expression of NGF receptor in the rat forebrain detected with in situ hybridization and immunohistochemistry. Brain Res Mol Brain Res 6:275-287.

Gonzalez MM, Aston-Jones G (2006) Circadian regulation of arousal: role of the noradrenergic locus coeruleus system and light exposure. Sleep 29:1327-1336.

Guldner FH, Wolff JR (1973) Neurono-glial synaptoid contacts in the median eminence of the rat: ultrastructure, staining properties and distribution on tanycytes. Brain Res 61:217-234.

Hagg T, Fass-Holmes B, Vahlsing HL, Manthorpe M, Conner JM, Varon S (1989) Nerve growth factor (NGF) reverses axotomy-induced decreases in choline acetyltransferase, NGF receptor and size of medial septum cholinergic neurons. Brain Res 505:29-38.

Hammerschlag R, Stone GC, Bolen FA, Lindsey JD, Ellisman MH (1982) Evidence that all newly synthesized proteins destined for fast axonal transport pass through the Golgi apparatus. J Cell Biol 93:568-575.

Hendry IA (1976) A method to correct adequately for the change in neuronal size when estimating neuronal numbers after nerve growth factor treatment. J Neurocytol 5:337-349.

Hendry IA, Campbell J (1976) Morphometric analysis of rat superior cervical ganglion after axotomy and nerve growth factor treatment. J Neurocytol 5:351-360.

Hochhaus F, Koehne P, Schaper C, Butenandt O, Felderhoff-Mueser U, RingMrozik E, Obladen M, Buhrer C (2001) Elevated nerve growth factor and neurotrophin-3 levels in cerebrospinal fluid of children with hydrocephalus. BMC Pediatr 1:2.

Hyland K (2008) Clinical utility of monoamine neurotransmitter metabolite analysis in cerebrospinal fluid. Clin Chem 54:633-641.

Jacobs JS, Miller MW (1999) Expression of nerve growth factor, p75, and the high affinity neurotrophin receptors in the adult rat trigeminal sys- 
tem: evidence for multiple trophic support systems. J Neurocytol 28:571-595.

Killer HE, Jaggi GP, Flammer J, Miller NR, Huber AR (2006) The optic nerve: a new window into cerebrospinal fluid composition? Brain 129:1027-1030.

Koevary SB, Lam V, Patsiopoulos G, Lake S (2003) Accumulation of porcine insulin in the rat brain and cerebrospinal fluid following ocular application. J Ocul Pharmacol Ther 19:377-384.

Koevary SB, Lam V, Patsiopoulos G (2004) Pharmacokinetics of insulin uptake by ocular tissues and the role of cerebrospinal fluid in optic nerve insulin accumulation following topical insulin application. Optometry 75:183-188.

Koh S, Oyler GA, Higgins GA (1989) Localization of nerve growth factor receptor messenger RNA and protein in the adult rat brain. Exp Neurol 106:209-221.

Kohara K, Kitamura A, Morishima M, Tsumoto T (2001) Activitydependent transfer of brain-derived neurotrophic factor to postsynaptic neurons. Science 291:2419-2423.

Lambiase A, Bonini S, Manni L, Ghinelli E, Tirassa P, Rama P, Aloe L (2002) Intraocular production and release of nerve growth factor after iridectomy. Invest Ophthalmol Vis Sci 43:2334-2340.

Lambiase A, Pagani L, Di Fausto V, Sposato V, Coassin M, Bonini S, Aloe L (2007) Nerve growth factor eye drop administrated on the ocular surface of rodents affects the nucleus basalis and septum: biochemical and structural evidence. Brain Res 1127:45-51.

Large TH, Weskamp G, Helder JC, Radeke MJ, Misko TP, Shooter EM, Reichardt LF (1989) Structure and developmental expression of the nerve growth factor receptor in the chicken central nervous system. Neuron 2:1123-1134.

Lehman M, Silver R (2000) CSF signaling in physiology and behavior. Prog Brain Res 125:415-433.

Levi-Montalcini R, Aloe L, Mugnaini E, Oesch F, Thoenen H (1975) Nerve growth factor induces volume increase and enhances tyrosine hydroxylase synthesis in chemically axotomized sympathetic ganglia of newborn rats. Proc Natl Acad Sci U S A 72:595-599.

Ludemann W, Berens von Rautenfeld D, Samii M, Brinker T (2005) Ultrastructure of the cerebrospinal fluid outflow along the optic nerve into the lymphatic system. Childs Nerv Syst 21:96-103.

Mashayekhi F, Salehi Z (2007) Infusion of anti-nerve growth factor into the cisternum magnum of chick embryo leads to decrease cell production in the cerebral cortical germinal epithelium. Eur J Neurol 14:181-186.

Mashayekhi F, Azari M, Moghadam LM, Yazdankhah M, Naji M, Salehi Z (2009) Changes in cerebrospinal fluid nerve growth factor levels during chick embryonic development. J Clin Neurosci 16:1334-1337.

Mufson EJ, Kroin JS, Sendera TJ, Sobreviela T (1999) Distribution and retrograde transport of trophic factors in the central nervous system: functional implications for the treatment of neurodegenerative diseases. Prog Neurobiol 57:451-484.

Nicholson C (1999) Signals that go with the flow. Trends Neurosci 22:143-145.

Nieuwenhuys R (2000) Comparative aspects of volume transmission, with sidelight on other forms of intercellular communication. Prog Brain Res 125:49-126.

Olpe HR, Jones RS (1983) The action of anticonvulsant drugs on the firing of locus coeruleus neurons: selective, activating effect of carbamazepine. Eur J Pharmacol 91:107-110.

Peters A, Palay SL, Webster Hd (1991) The fine structure of the nervous system: neurons and their supporting cells, Ed 3. New York: Oxford UP.

Purves D (1988) Body and brain: a trophic theory of neural connections. Cambridge, MA: Harvard UP.

Racagni G, Brunello N (1984) Transynaptic mechanisms in the action of antidepressant drugs. Trends Pharmacol Sci 5:527-531.
Rodman HR, Karten HJ (1995) Laminar distribution and sources of catecholaminergic input to the optic tectum of the pigeon (Columba livia). J Comp Neurol 359:424-442.

Rodriguez EM, Blazquez JL, Pastor FE, Pelaez B, Pena P, Peruzzo B, Amat P (2005) Hypothalamic tanycytes: a key component of brain-endocrine interaction. Int Rev Cytol 247:89-164.

Scott DE, Dudley GK, Knigge KM (1974) The ventricular system in neuroendocrine mechanisms. II. In vivo Monoamine transport by ependyma of the median eminence. Cell Tissue Res 154:1-16.

Shen JY, Kelly DE, Hyman S, McComb JG (1985) Intraorbital cerebrospinal fluid outflow and the posterior uveal compartment of the hamster eye. Cell Tissue Res 240:77-87.

Smialowska M, Bal-Klara A, Smialowski A (1988) Chronic imipramine diminishes the nuclear size of neurons in the locus coeruleus and cingular cortex but not in the hippocampus of the rat brain. Neuroscience 26:803-807.

Valentino RJ, Van Bockstaele E (2008) Convergent regulation of locus coeruleus activity as an adaptive response to stress. Eur J Pharmacol 583:194-203.

Van der Zee CE, Rashid K, Le K, Moore KA, Stanisz J, Diamond J, Racine RJ, Fahnestock M (1995) Intraventricular administration of antibodies to nerve growth factor retards kindling and blocks mossy fiber sprouting in adult rats. J Neurosci 15:5316-5323.

Vigh B, Vigh-Teichmann I (1998) Actual problems of the cerebrospinal fluid-contacting neurons. Microsc Res Tech 41:57-83.

von Bartheld CS (2001) Tracing with radiolabeled neurotrophins. Methods Mol Biol 169:195-216.

von Bartheld CS, Bothwell M (1992) Development and distribution of noradrenergic and cholinergic neurons and their trophic phenotypes in the avian ceruleus complex and midbrain tegmentum. J Comp Neurol 320:479-500.

von Bartheld CS, Fritzsch B (2006) Comparative analysis of neurotrophin receptors and ligands in vertebrate neurons: tools for evolutionary stability or changes in neural circuits? Brain Behav Evol 68:157-172.

von Bartheld CS, Meyer DL (1990) Paraventricular organ of the lungfish Protopterus dolloi: morphology and projections of CSF-contacting neurons. J Comp Neurol 297:410-434.

von Bartheld CS, Schober A, Kinoshita Y, Williams R, Ebendal T, Bothwell M (1995) Noradrenergic neurons in the locus coeruleus of birds express TrkA, transport NGF, and respond to NGF. J Neurosci 15:2225-2239.

von Bartheld CS, Byers MR, Williams R, Bothwell M (1996a) Anterograde transport of neurotrophins and axodendritic transfer in the developing visual system. Nature 379:830-833.

von Bartheld CS, Williams R, Lefcort F, Clary DO, Reichardt LF, Bothwell M (1996b) Retrograde transport of neurotrophins from the eye to the brain in chick embryos: roles of the p75NTR and trkB receptors. J Neurosci 16:2995-3008.

von Bartheld CS, Wang XX, Butowt R (2001) Anterograde axonal transport, transcytosis, and recycling of neurotrophic factors: the concept of trophic currencies in neural networks. Mol Neurobiol 24:1-28.

Wang XX, Butowt R, von Bartheld CS (2003) Presynaptic neurotrophin-3 increases the number of tectal synapses, vesicle density, and number of docked vesicles in chick embryos. J Comp Neurol 458:62-77.

Xia YX, Ikeda T, Xia XY, Ikenoue T (2000) Differential neurotrophin levels in cerebrospinal fluid and their changes during development in newborn rat. Neurosci Lett 280:220-222.

Yu J, Xie LY, Abou-Samra AB (1996) Molecular cloning of a type A chicken corticotropin-releasing factor receptor with high affinity for urotensin I. Endocrinology 137:192-197.

Zarow C, Lyness SA, Mortimer JA, Chui HC (2003) Neuronal loss is greater in the locus coeruleus than nucleus basalis and substantia nigra in Alzheimer and Parkinson diseases. Arch Neurol 60:337-341. 